This item was submitted to Loughborough's Research Repository by the author.

Items in Figshare are protected by copyright, with all rights reserved, unless otherwise indicated.

\title{
Analysis of mutually incoherent symmetrical illumination for electronic speckle pattern shearing interferometry
}

PLEASE CITE THE PUBLISHED VERSION

PUBLISHER

(C) Taylor \& Francis

LICENCE

CC BY-NC-ND 4.0

\section{REPOSITORY RECORD}

Roman, Juan F., Vicente Moreno, Jon N. Petzing, and John R. Tyrer. 2019. "Analysis of Mutually Incoherent Symmetrical Illumination for Electronic Speckle Pattern Shearing Interferometry". figshare. https://hdl.handle.net/2134/3635. 
This item was submitted to Loughborough's Institutional Repository by the author and is made available under the following Creative Commons Licence conditions.

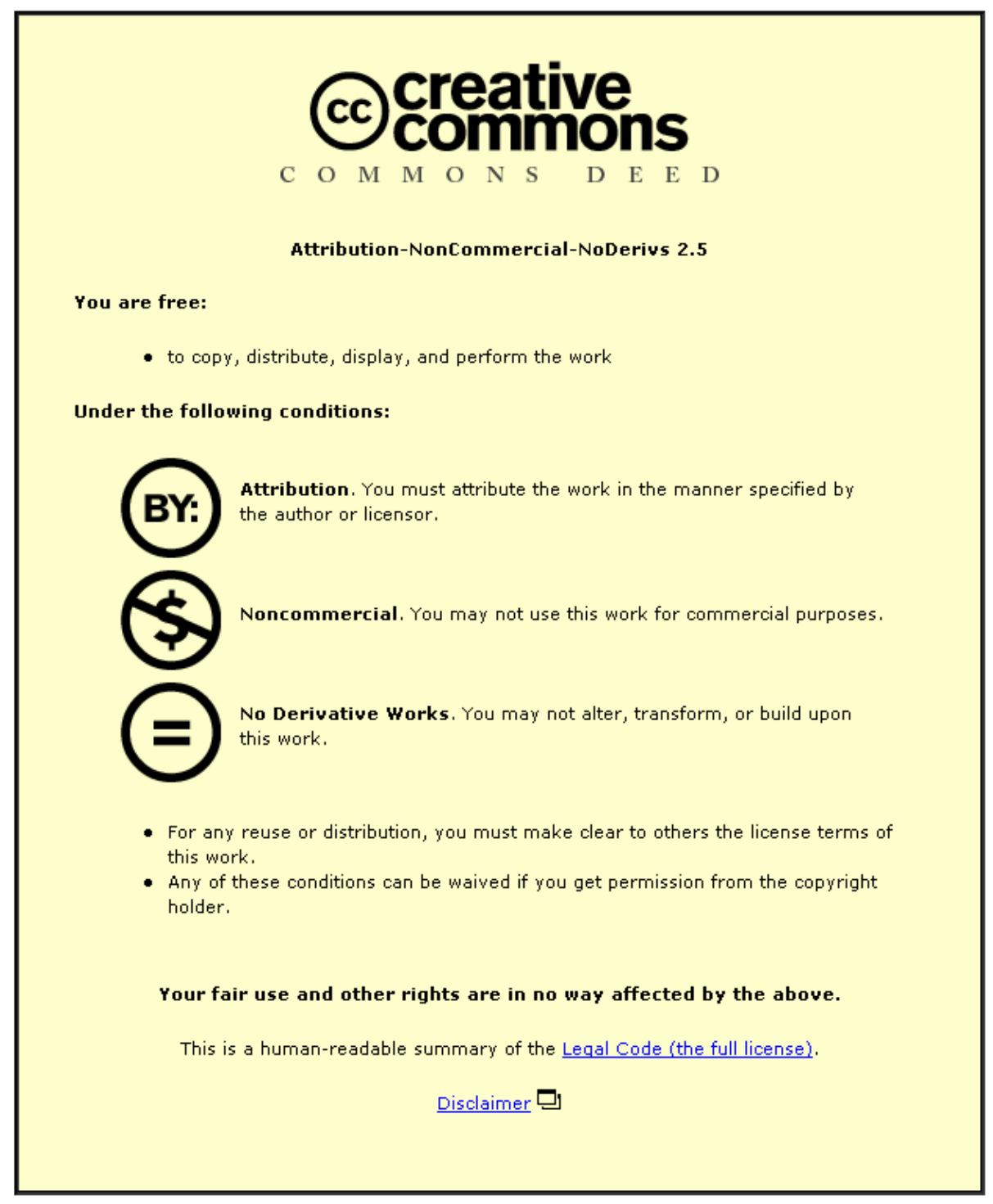

For the full text of this licence, please go to: http://creativecommons.org/licenses/by-nc-nd/2.5/ 
5 Analysis of mutually incoherent symmetrical illumination for electronic speckle pattern shearing interferometry JUAN F. ROMÁN * V, VICENTE MORENO $\delta$, JON N. PETZING $\ddagger$ and JOHN R. TYRER $\ddagger$

$11 \sqrt{ } \quad$ Bayer Diagnostics, Sudbury, CO10 2XQ, Suffolk, UK.

12 juan.roman.jr@bayer.co.uk

13 Faculty of Physics, University of Santiago de Compostela, 15782, 14 Santiago de Compostela, Spain.

$15 \ddagger$ Wolfson School of Mechanical and Manufacturing Engineering, 16 Loughborough University, Loughborough, Leics., LE11 3TU, UK. 
3 The authors designed a speckle shearing interferometer using symmetrical 4 mutually incoherent illumination, in an effort to provide measurements of in-plane 5 strain. It is presented the analysis of the sensitivity to displacement and strain of this 6 interferometer, together with the analysis of the optical phase extraction of the 7 resultant fringe pattern. This interferometer is an improvement on previous designs as 8 it provides information of the in-plane strain separated from components of the 9 displacement. Experimental results show the fringe patterns in support of the 10 theoretical analysis.

14 Keywords: Speckle, Interferometry, Strain, ESPI, ESPSI 


\section{INTRODUCTION}

2 Optical metrology techniques based on speckle provide measurements of

3 displacement and strain. They bring the advantages of being a whole-field

4 measurement, non-contact and in real-time [1]. Displacement and strain can be

5 divided into their orthogonal components, the Out-Of-Plane (OOP) component and

6 the two X and Y In-Plane (IP) components. Electronic Speckle Pattern Interferometry

7 (ESPI) is used for the measurement of displacement in the three orthogonal axes,

8 allowing the independent extraction of the two IP and the one OOP displacement 9 components [2].

Electronic Speckle Pattern Shearing Interferometry (ESPSI) provides a

11 measurement of the first spatial derivatives of the displacement, these being related to

12 mechanical strain $[3,4]$. This measurement is of great practical interest since

13 engineering designs can generally cope with microscopic displacements of their

14 components, but must avoid unwanted accumulation of strain in the mechanisms.

15 Several speckle shearing interferometers can discretely measure the OOP spatial

16 derivative component [4-8]. However, this is not the case for discrete measurement of

17 the in-plane spatial derivative components, where ESPSI has technical difficulties.

The in-plane spatial derivative terms have been extracted using aperture based

19 designs and Fourier plane analysis [9] but these systems have yet to be demonstrated

20 as real-time instruments. Alternatively, extraction of the in-plane spatial derivative

21 terms has been demonstrated using sequential measurement, changing illumination

22 angles between each data set, [4, 10-12]. Whilst this approach does work, if the

23 deformation of the object under study changes between each of the sequential

24 measurements, as it is often the case with real-life engineering problems, then the 
1 technique will not produce the correct results. A solution to this issue has been 2 proposed [13], with a shearing interferometer utilising three wavelengths for

3 illumination, but the complexity of the system should perhaps not be underestimated.

Direct measurements of in-plane strain components have been presented but

5 under special circumstances, such as plane stress or plane strain conditions [14].

6 Further work has suggested that the use of dual or simultaneous illumination

7 wavefronts may allow direct analysis of in-plane components for arbitrary objects $8 \quad[15]$.

Speckle interferometers using more than one illuminating beam generate complex interference patterns whose analysis requires careful understanding of the

11 optical properties of speckle, as well as analysis of the geometry of object and image.

12 The standard notation used to describe speckle shearing interferometer output $[1,2]$,

13 reduces the expression for the interference of the optical wavefronts at the observation 14 plane, to a cosine expression that contains the addition of phase delays. However, this 15 does not take into account the relative spatial correlations of the speckle patterns 16 scattered from different incident beams or scattered from different areas of the object, 17 separated by the lateral shear. Furthermore, for a dual beam system, the standard 18 notation used for speckle shearing interferometry does not provide indicators for 19 which wavefronts or which illuminating beams interact, after the lateral shift of the 20 images, or after the absolute value subtraction of the image patterns.

21 The approach taken in our work has been to modify the expression describing 22 the speckle interference pattern so that it is separated into several intensity terms, each 23 one labelled according to which illuminating wavefront contributes to it. These labels 
1 take into account the polarisation state of each wavefront, in order to indicate which

2 ones cause interference and which others will just add together their intensities.

Prior work has used the notation described above to deal with dual beam

4 mutually coherent speckle shearing interferometers [16, 23]. The same notation is

5 utilised here to study the possibility of a dual beam mutually incoherent illumination

6 interferometer for deformation analysis. The theoretical analysis for the new

7 interferometer is presented in this work, together with the initial experimental results 8 that support the predictions.

9

\section{THEORETICAL ANALYSIS}

11 The proposed interferometer uses two mutually incoherent and symmetrically incident

12 beams, beams Left (L) and Right (R), to illuminate the object, Figure 1. The lateral

13 shifting of the images is performed by means of a Michelson design with two non-

14 perpendicular mirrors (A and $\mathrm{B}$ ), and the optical axis of the CCD camera bisects the 15 angle made by the two laser illumination beams. It should also be noted that, as would 16 be expected with a Michelson based optical system, the theoretical development has

17 many initial similarities with existing speckle pattern interferometry theory $[1,23]$, 18 although modified and expanded to take into account the nature of simultaneous 19 incoherent illumination.

The analysis of the interferometer starts with the labelling of each component

21 of the optical set-up: if we denote by LA, LB, RA, RB the amplitudes, phase included, 22 of the wavefronts arriving to a point $(\mathrm{x}, \mathrm{y})$ on the image plane, the intensity registered 
1 by the sensors of the camera will be given by eq. [1], where $L A=\|L A\| \cdot e^{i \phi_{L A}}$ and $2 \quad \mathrm{LA}^{*}=\|\mathrm{LA}\| \cdot \mathrm{e}^{-\mathrm{i} \phi_{\mathrm{LA}}}$ :

$$
\mathrm{I}(\mathrm{x}, \mathrm{y})=(\mathrm{LA}+\mathrm{LB}) \cdot(\mathrm{LA}+\mathrm{LB}) *+(\mathrm{RA}+\mathrm{RB}) \cdot(\mathrm{RA}+\mathrm{RB}) *
$$

The beams $\mathrm{L}$ and $\mathrm{R}$ are not coherent between themselves and thus can not

5 make interference with each other. Different experimental conditions can simulate this

6 case: set-ups with two beams with their polarisations orthogonal to each other, as

7 shown in Figure 1, set-ups where each beam comes from a different laser source, or

8 finally set-ups with two beams equally polarised but with a difference of optical path

9 bigger than the coherence length of the laser source. The case of two beams with

10 different wavelengths is not considered on this analysis.

In eq. [1], $(\mathrm{x}, \mathrm{y})$ represents any point on the CCD camera sensing area, $\mathrm{I}(\mathrm{x}, \mathrm{y})$ is

12 the intensity of light registered at that point, and the * sign represents the conjugated

13 wave. The product [1] results in eq. [2]:

$$
\begin{gathered}
\mathrm{I}(\mathrm{x}, \mathrm{y})=\mathrm{LA} \cdot \mathrm{LA} *+\mathrm{LB} \cdot \mathrm{LB} *+\mathrm{LA} \cdot \mathrm{LB} *+\mathrm{LB} \cdot \mathrm{LA}^{*}+ \\
\mathrm{RA} \cdot \mathrm{RA} *+\mathrm{RB} \cdot \mathrm{RB} *+\mathrm{RA} \cdot \mathrm{RB} *+\mathrm{RB} \cdot \mathrm{RA} *
\end{gathered}
$$

15 where the terms of the addition $\mathrm{LA} \cdot \mathrm{LA}^{*}, \mathrm{LB} \cdot \mathrm{LB} *, \mathrm{RA} \cdot \mathrm{RA}^{*}$ and $\mathrm{RB} \cdot \mathrm{RB}^{*}$ represent

16 the intensity of the beams LA, LB, RA, RB respectively, eq. [3]:

$$
\mathrm{LA} \cdot \mathrm{LA}^{*}=\|\mathrm{LA}\| \cdot \mathrm{e}^{\mathrm{i} \phi_{\mathrm{LA}}} \cdot\|\mathrm{LA}\| \cdot \mathrm{e}^{-\mathrm{i} \phi_{\mathrm{LA}}}=\|\mathrm{LA}\|^{2}
$$


1 where IILA\| is the modulus of the amplitude of the electric field associated to the light

2 beam LA, $\phi_{\mathrm{LA}}$ represents its phase and $\|\mathrm{LA}\|^{2}$ its intensity. The phase $\phi_{\mathrm{LA}}$ is a random

3 value that depends on the roughness of the surface on that particular point of the

4 object. Though this is a random value, it will remain constant as long as the surface is

5 not affected or eroded.

6 Applying similar calculations to the rest of the terms in eq [2], the result will be equations [4], [5] and [6]:

8

9

$$
L B \cdot \mathbf{L B}^{*}=\|\mathrm{LB}\| \cdot \mathrm{e}^{\mathrm{i} \phi_{\mathrm{LB}}} \cdot\|\mathrm{LB}\| \cdot \mathrm{e}^{-\mathrm{i \phi _{ \textrm {LB } }}}=\|\mathrm{LB}\|^{2}
$$

$$
\mathrm{RA} \cdot \mathrm{RA}^{*}=\|\mathrm{RA}\| \cdot \mathrm{e}^{\mathrm{i} \phi_{\mathrm{RA}}} \cdot\|\mathrm{RA}\| \cdot \mathrm{e}^{-\mathrm{i} \phi_{\mathrm{RA}}}=\|\mathrm{RA}\|^{2}
$$
the object plane, scattered from the incident beams $\mathrm{L}$ and $\mathrm{R}$ respectively and reflected by mirror $\mathrm{A}$, making incidence onto point $(\mathrm{x}, \mathrm{y})$ of the CCD plane. Analogously, LB and $\mathrm{RB}$ will be the light wavefronts scattered from point $(\mathrm{x}+\delta \mathrm{x}, \mathrm{y})$ on the object plane, reflected by mirror B (tilted) and incident onto the same point (x,y) on the CCD plane. The tilting on mirror B introduces the lateral shear $\delta x$.

The rest of the terms in $\mathrm{I}(\mathrm{x}, \mathrm{y})$ can be calculated using the same notation for the amplitude and phase, eq. [7] and eq. [8]:

$$
\begin{aligned}
\mathrm{RA} \cdot \mathrm{RB}^{*}+\mathrm{RB} \cdot \mathrm{RA}^{*} & =\|\mathrm{RA}\| \cdot\|\mathrm{RB}\| \cdot \mathrm{e}^{\mathrm{i} \phi_{\mathrm{RA}}} \cdot \mathrm{e}^{-\mathrm{i} \phi_{\mathrm{RB}}}+\|\mathrm{RA}\| \cdot\|\mathrm{RB}\| \cdot \mathrm{e}^{-\mathrm{i} \phi_{\mathrm{RA}}} \cdot \mathrm{e}^{\mathrm{i} \phi_{\mathrm{RB}}}= \\
& =\|\mathrm{RA}\| \cdot\|\mathrm{RB}\| \cdot\left\{\mathrm{e}^{\mathrm{i}\left(\phi_{\mathrm{RA}}-\phi_{\mathrm{RB}}\right)}+\mathrm{e}^{\mathrm{i}\left(\phi_{\mathrm{RB}}-\phi_{\mathrm{RA}}\right)}\right\} \\
& =\|\mathrm{RA}\| \cdot\|\mathrm{RB}\| \cdot 2 \cdot \operatorname{Cos}\left(\phi_{\mathrm{RA}}-\phi_{\mathrm{RB}}\right)
\end{aligned}
$$


The final result is expressed by eq. [9]:

$$
\begin{aligned}
\mathrm{I}(\mathrm{x}, \mathrm{y}) & =\|\mathrm{LA}\|^{2}+\|\mathrm{LB}\|^{2}+\|\mathrm{RA}\|^{2}+\|\mathrm{RB}\|^{2} \\
& +\|\mathrm{RA}\| \cdot\|\mathrm{RB}\| \cdot 2 \cdot \operatorname{Cos}\left(\phi_{\mathrm{RA}}-\phi_{\mathrm{RB}}\right) \\
& +\|\mathrm{LA}\| \cdot\|\mathrm{LB}\| \cdot 2 \cdot \operatorname{Cos}\left(\phi_{\mathrm{LA}}-\phi_{\mathrm{LB}}\right)
\end{aligned}
$$

This equation represents the intensity of light at the arbitrary point $(\mathrm{x}, \mathrm{y})$ on the

5 CCD camera sensing plane, before the object undergoes any alteration or deformation.

We assume that the object deformation or displacement is smaller than the average speckle grain size, an assumption generally made for all the correlation speckle interferometers. Hence the intensity of each beam at the point $(\mathrm{x}, \mathrm{y})$ will be the same before and after the changes on the object, as expressed by eq. [10]:

$$
\|\mathrm{LA}\|_{\text {after }}=\|\mathrm{LA}\|_{\text {before }}
$$
As previously discussed, the intensity and phase of light arising from mirrors

$12 \mathrm{~A}$ and $\mathrm{B}$ are different due to the different angle of illumination, the image shift 13 introduced by mirror B and the different deformations experimented by the object on 14 points $(\mathrm{x}, \mathrm{y})$ and $(\mathrm{x}+\delta \mathrm{x}, \mathrm{y})$ :

$$
\|\mathrm{LA}\| \neq\|\mathrm{RA}\| \neq\|\mathrm{LB}\| \neq\|\mathrm{RB}\|
$$

$$
\begin{gathered}
\phi_{\mathrm{RA}} \neq \phi_{\mathrm{RB}} \\
\left(\phi_{\mathrm{LA}}-\phi_{\mathrm{LB}}\right)^{\mathrm{Before}} \neq\left(\phi_{\mathrm{LA}}-\phi_{\mathrm{LB}}\right)^{\mathrm{After}}
\end{gathered}
$$


The phase changes introduced by the deformation or displacement of the

3 object will change the recorded intensity pattern. Both in-plane and out-of-plane

4 displacements introduce phase changes that modify the resultant speckled pattern of

5 intensity. According to Table 1, the intensity registered after the displacement will be

6 expressed by eq. [14]:

7

$$
\begin{aligned}
& \mathrm{I}(\mathrm{x}, \mathrm{y})_{\mathrm{CCD}}^{\mathrm{After}}=\|\mathrm{LA}\|^{2}+\|\mathrm{LB}\|^{2}+\|\mathrm{RA}\|^{2}+\|\mathrm{RB}\|^{2} \\
& +\|\mathrm{RA}\| \cdot\|\mathrm{RB}\| \cdot 2 \cdot \operatorname{Cos}\left(\begin{array}{l}
{\left[\phi_{\mathrm{RA}}+\frac{2 \pi}{\lambda}(-\mathrm{u} \cdot \operatorname{Sin} \theta-\mathrm{w} \cdot(1+\operatorname{Cos} \theta))\right]-} \\
\left.\left[\phi_{\mathrm{RB}}+\frac{2 \pi}{\lambda}(-(\mathrm{u}+\delta \mathrm{u}) \cdot \operatorname{Sin} \theta-(\mathrm{w}+\delta \mathrm{w}) \cdot(1+\operatorname{Cos} \theta))\right]\right)
\end{array}\right) \\
& +\|\mathrm{LA}\| \cdot\|\mathrm{LB}\| \cdot 2 \cdot \operatorname{Cos}\left(\begin{array}{l}
{\left[\phi_{\mathrm{LA}}+\frac{2 \pi}{\lambda}(\mathrm{u} \cdot \operatorname{Sin} \theta-\mathrm{w} \cdot(1+\operatorname{Cos} \theta))\right]-} \\
\left.\left[\phi_{\mathrm{LB}}+\frac{2 \pi}{\lambda}((\mathrm{u}+\delta \mathrm{u}) \cdot \operatorname{Sin} \theta-(\mathrm{w}+\delta \mathrm{w}) \cdot(1+\operatorname{Cos} \theta))\right]\right)
\end{array}\right)
\end{aligned}
$$

$$
\begin{aligned}
& \mathrm{I}(\mathrm{x}, \mathrm{y})_{\mathrm{CCD}}^{\mathrm{After}}=\|\mathrm{LA}\|^{2}+\|\mathrm{LB}\|^{2}+\|\mathrm{RA}\|^{2}+\|\mathrm{RB}\|^{2} \\
& +\|\mathrm{LA}\| \cdot\|\mathrm{LB}\| \cdot 2 \cdot \operatorname{Cos}[\phi_{\mathrm{LA}}-\phi_{\mathrm{LB}}+\underbrace{\frac{2 \pi}{\lambda}(-\delta \mathrm{u} \cdot \operatorname{Sin} \theta+\delta \mathrm{w}(1+\operatorname{Cos} \theta))}_{\Delta_{1}}] \\
& +\|\mathrm{RA}\| \cdot\|\mathrm{RB}\| \cdot 2 \cdot \operatorname{Cos}[\phi_{\mathrm{RA}}-\phi_{\mathrm{RB}}+\underbrace{\frac{2 \pi}{\lambda}(\delta \mathrm{u} \cdot \operatorname{Sin} \theta+\delta \mathrm{w}(1+\operatorname{Cos} \theta))}_{\Delta_{2}}]
\end{aligned}
$$

Beams $\mathrm{L}$ and $\mathrm{R}$ are incoherent with each other and thus the interference terms

11 do not include crossed terms containing both. This reduces the number of interference terms and simplifies the resultant moiré inter-crossing of speckle fringe patterns. That 
1 represents an improvement from the design with two mutually coherent symmetrical 2 beams [23]. The images recorded before and after the deformation of the object are

4 subtracted in intensity pixel by pixel. The resultant image will have dark speckle

5 fringes on the places of the object where $\mathrm{I}^{\text {before }}(\mathrm{x}, \mathrm{y})=\mathrm{I}^{\mathrm{after}}(\mathrm{x}, \mathrm{y})$. In order for this to

6 happen the two interference terms in eq. [15], $\Delta_{1}$ and $\Delta_{2}$, must be equal to an even

7 number of times $\pi$ :

8

9

11 shearing $\delta x$ between the images is small, these expressions may be rewritten in a

$$
\begin{aligned}
& \Delta_{1}=\frac{2 \pi}{\lambda}(-\delta \mathrm{u} \cdot \operatorname{Sin} \theta+\delta \mathrm{w}(1+\operatorname{Cos} \theta))=2 \cdot \mathrm{m}_{1} \cdot \pi \\
& \Delta_{2}=\frac{2 \pi}{\lambda}(\delta \mathrm{u} \cdot \operatorname{Sin} \theta+\delta \mathrm{w}(1+\operatorname{Cos} \theta))=2 \cdot \mathrm{m}_{2} \cdot \pi
\end{aligned}
$$

where $m_{1}$ and $m_{2}$ are integer numbers and represent the fringe order. As the lateral differential form as:

$$
\begin{aligned}
& \Delta_{1}=\frac{2 \pi}{\lambda} \cdot\left[-\left(\frac{\partial \mathrm{u}}{\partial \mathrm{x}}\right) \cdot \delta \mathrm{x} \cdot \operatorname{Sin} \theta+\left(\frac{\partial \mathrm{w}}{\partial \mathrm{x}}\right) \cdot \delta \mathrm{x} \cdot(1+\operatorname{Cos} \theta)\right]=2 \cdot \mathrm{m}_{1} \cdot \pi \\
& \Delta_{2}=\frac{2 \pi}{\lambda} \cdot\left[\left(\frac{\partial \mathrm{u}}{\partial \mathrm{x}}\right) \cdot \delta \mathrm{x} \cdot \operatorname{Sin} \theta+\left(\frac{\partial \mathrm{w}}{\partial \mathrm{x}}\right) \cdot \delta \mathrm{x} \cdot(1+\operatorname{Cos} \theta)\right]=2 \cdot \mathrm{m}_{2} \cdot \pi
\end{aligned}
$$

Only on the points $(\mathrm{x}, \mathrm{y})$ of the image where both conditions, equations [18] and [19], are simultaneously satisfied the result of the image subtraction will provide a dark fringe. As the two equations must be satisfied simultaneously, their addition and subtraction must be a condition as well for the observation of the fringes. After some calculation, the final results are: 


$$
2 \cdot\left(\frac{\partial u}{\partial x}\right) \cdot \delta x \cdot \operatorname{Sin} \theta=m^{\prime \prime} \cdot \lambda
$$

The resultant pattern is a moiré-like pattern resultant from the intersection of two patterns: the fringe pattern of the pure out-of-plane strain, eq. [20], inter-crossing

5 with the fringe pattern of the pure in-plane strain, eq. [21]. The in-plane component of

6 the strain is completely separated from the out-of-plane component, in a moiré-like

7 pattern. The extraction of the out-of-plane component of the strain can be done 8 independently by means of a second camera and source recording only the out-of9 plane component, in a typical perpendicular illumination speckle shearing set-up.

11 new in itself, with authors having previously presented photographic based systems 12 using Fourier analysis [17, 18], to examine out-of-plane and in-plane terms. However, 13 the intention in our work here is to explore optical configurations which result directly 14 in real-time in-plane derivative systems, with the potential for quantitative evaluation.

\section{OPTICAL PHASE EXTRACTION}

Optical phase information extraction provides the tool for eventual generation of quantified data from the instrumentation. By introducing a controlled optical phase

19 shift within the interferometer, it is possible to perform the phase stepping [19] of the resultant speckle pattern and fringe pattern. 
2 introducing a phase step in one of the illuminating beams or introducing a phase step

3 within the Michelson shearing-head.

If a phase-step is introduced in one of the illuminating beams, this will not

5 result in a phase stepping of the speckle fringe pattern: the two illuminating beams are

6 already incoherent to each other and an additional phase shift between them can not

7 introduce any phase difference that will arise to a change in the speckle pattern. Hence

8 the only option is introducing the phase step within the Michelson shearing-head.

In order to do that, one of the mirrors must be pushed forward and backwards

10 by means of a piezo-electric actuator, introducing a phase-shift within the laterally

11 sheared images. To analyse the effect of this type of phase-shift it can be assumed for

12 the sake of argument that the piezo actuator is fitted to mirror B. The phase-shift

13 introduced results in $\phi_{\mathrm{LB}}$ changing into $\phi_{\mathrm{LB}}+\phi^{\prime}$ and $\phi_{\mathrm{RB}}$ into $\phi_{\mathrm{RB}}+\phi$, generating a

14 speckle intensity change after the displacement or deformation of the object, as

15 described by eq. [22]:

$$
\mathrm{I}(\mathrm{x}, \mathrm{y})_{\mathrm{CCD}}^{\mathrm{After}}=\|\mathrm{LA}\|^{2}+\|\mathrm{LB}\|^{2}+\|\mathrm{RA}\|^{2}+\|\mathrm{RB}\|^{2}
$$

$16+\|\mathrm{RA}\| \cdot\|\mathrm{RB}\| \cdot 2 \cdot \operatorname{Cos}\left(\begin{array}{l}{\left[\phi_{\mathrm{RA}}+\frac{2 \pi}{\lambda}(-\mathrm{u} \cdot \operatorname{Sin} \theta-\mathrm{w} \cdot(1+\operatorname{Cos} \theta))\right]-} \\ {\left[\left(\phi_{\mathrm{RB}}+\phi^{\prime}\right)+\frac{2 \pi}{\lambda}(-(\mathrm{u}+\delta \mathrm{u}) \cdot \operatorname{Sin} \theta-(\mathrm{w}+\delta \mathrm{w}) \cdot(1+\operatorname{Cos} \theta))\right]}\end{array}\right)$

$+\|\mathrm{LA}\| \cdot\|\mathrm{LB}\| \cdot 2 \cdot \operatorname{Cos}\left(\begin{array}{l}{\left[\phi_{\mathrm{LA}}+\frac{2 \pi}{\lambda}(\mathrm{u} \cdot \operatorname{Sin} \theta-\mathrm{w} \cdot(1+\operatorname{Cos} \theta))\right]-} \\ {\left[\left(\phi_{\mathrm{LB}}+\phi^{\prime}\right)+\frac{2 \pi}{\lambda}((\mathrm{u}+\delta \mathrm{u}) \cdot \operatorname{Sin} \theta-(\mathrm{w}+\delta \mathrm{w}) \cdot(1+\operatorname{Cos} \theta))\right]}\end{array}\right)$

17 This expression leads to eq. [23]: 


$$
\begin{aligned}
& \mathrm{I}(\mathrm{x}, \mathrm{y})_{\mathrm{CCD}}^{\mathrm{After}}=\|\mathrm{LA}\|^{2}+\|\mathrm{LB}\|^{2}+\|\mathrm{RA}\|^{2}+\|\mathrm{RB}\|^{2} \\
& +\|\mathrm{LA}\| \cdot\|\mathrm{LB}\| \cdot 2 \cdot \operatorname{Cos}[\phi_{\mathrm{LA}}-\phi_{\mathrm{LB}} \underbrace{-\phi^{\prime}+\frac{2 \pi}{\lambda}(-\delta \mathrm{u} \cdot \operatorname{Sin} \theta+\delta \mathrm{w}(1+\operatorname{Cos} \theta))}_{\Delta_{1}^{\prime}}] \\
& +\|\mathrm{RA}\| \cdot\|\mathrm{RB}\| \cdot 2 \cdot \operatorname{Cos}[\phi_{\mathrm{RA}}-\phi_{\mathrm{RB}} \underbrace{-\phi^{\prime}+\frac{2 \pi}{\lambda}(\delta \mathrm{u} \cdot \operatorname{Sin} \theta+\delta \mathrm{w}(1+\operatorname{Cos} \theta))}_{\Delta_{2}^{\prime}}]
\end{aligned}
$$

2 where the interference terms $\Delta_{1}$ ' and $\Delta_{2}$ ' include now the phase shift $\phi$ ', equations $3[24]$ and [25]:

$$
\Delta_{1}^{\prime}=\frac{2 \pi}{\lambda} \cdot\left[-\left(\frac{\partial \mathrm{u}}{\partial \mathrm{x}}\right) \cdot \delta \mathrm{x} \cdot \operatorname{Sin} \theta+\left(\frac{\partial \mathrm{w}}{\partial \mathrm{x}}\right) \cdot \delta \mathrm{x} \cdot(1+\operatorname{Cos} \theta)\right]-\phi^{\prime}=2 \cdot \mathrm{m}_{1} \cdot \pi
$$

$$
\Delta_{2}^{\prime}=\frac{2 \pi}{\lambda} \cdot\left[\left(\frac{\partial \mathrm{u}}{\partial \mathrm{x}}\right) \cdot \delta \mathrm{x} \cdot \operatorname{Sin} \theta+\left(\frac{\partial \mathrm{w}}{\partial \mathrm{x}}\right) \cdot \delta \mathrm{x} \cdot(1+\operatorname{Cos} \theta)\right]-\phi^{\prime}=2 \cdot \mathrm{m}_{2} \cdot \pi
$$

6 where $m_{1}, m_{2}$ are integer numbers that indicate the fringe order. After some

7 calculations these two equations lead to:

8

9

$$
2 \cdot\left(\frac{\partial u}{\partial x}\right) \cdot \delta x \cdot \operatorname{Sin} \theta-\frac{\phi^{\prime}}{2 \cdot \pi}=m^{\prime \prime} \cdot \lambda
$$
The resultant pattern is a moiré-like pattern obtained from the intersection of

11 two patterns: the phase shifted fringe pattern of the pure out-of-plane strain, eq. [26], 12 inter-crossing with the phase shifted fringe pattern of the pure in-plane strain, eq. [27]. As a summary, in this interferometer the introduction of phase stepping in one of the illuminating beams does not have any effect on the phase-stepping of the resultant 
1 fringe pattern. On the other hand, the introduction of phase stepping within the

2 Michelson shearing head results in a phase shift on both components of the moiré

3 pattern, the out-of-plane strain and the in-plane strain. This property of phase stepping

4 followed by a phase unwrapping process may be used to extract optical phase

5 information, and hence quantitative data, from this interferometer. 


\section{4. EXPERIMENTAL RESULTS}

2 The theoretical analysis predicts that the dual beam mutually incoherent

3 interferometer will produce moiré patterns consistent of the inter-crossing of the out-

4 of-plane strain pattern with the in-plane strain pattern. The goal of the

5 experimentation was to demonstrate that the interferometer actually follows that

6 prediction. For that purpose, and using the same specimen and experimental

7 conditions, we first obtained in-plane deformation data by means of in-plane ESPI and

8 numerically differentiated it to obtain a map of in-plane strain. In order to corroborate

9 this data we then applied photoelasticity techniques to obtain a qualitative distribution

10 of in-plane strain across the sample. Finally we obtained the map of out-of-plane

11 strain by means of conventional OOP-ESPSI. The OOP-strain fringe patterns obtained by this technique when overlapped with the IP-strain distribution numerically obtained from the IP-deformation data, produce a moiré fringe pattern that was

14 successfully replicated by means of the new dual beam mutually incoherent ESPSI 15 interferometer.

The experiments were performed on a Brazilian Disk specimen, or split

17 cylinder test, under compressive loading. This type of specimen consists of a flat disk compressed along its equator and has traditionally been used with techniques such as

19 photoelasticity, moiré interferometry or holographic interferometry [20-21]. A 75mm

20 diameter and $6 \mathrm{~mm}$ thick Brazilian disk was manufactured from an Araldite $^{\mathrm{TM}}$ sheet,

21 and was compressed using a test rig linked to a hydraulic DH-Budenburg dead-weight 22 tester. This provided forces up to $5000 \mathrm{~N}$, with a precision of $\pm 1 \mathrm{~N}$.

The interferometer used a 50mWatt Nd-YAG laser (wavelength $\lambda=532 \mathrm{~nm}$ ), with a 24 coherence length in excess of $10 \mathrm{~m}$. The camera used to record the images was a CCD 
1 Pulnix TM-9701 linked to a MuTech Corporation image processing board. Optical

2 phase-stepping was introduced by means of a piezoelectric actuator by Piezo-Systems 3 Jena.

\subsection{In plane ESPI analysis of the Brazilian disk}

6 The in-plane deformation map on the Brazilian disk under compression was measured

7 by means of an in-plane ESPI set-up. The set of figures, Figures 2 a), b) and c), shows

8 a set of phase-stepped fringe patterns of horizontal illumination ESPI for the Brazilian

9 disk under a force of $17.5 \pm 0.1 \mathrm{Kg}$-Force (171.6 \pm 1 Newton). The first Figure 2.a)

10 corresponds to the subtraction between the reference image and the image of the

11 deformed object, and the other two images, Figure 2.b) and 2.c), correspond to the

$12 \pm 2 \pi / 3$ phase-stepped fringe patterns respectively.

The fringe located on the left hand side of the disk corresponds to the order $\mathrm{m}=0$, the part of the disk pressed against the stop that does not perform any displacement. The fringe pattern indicates that the displacement distributes evenly across the surface of the disk, with an expected increase in the spatial frequency of the

17 fringes at the contact points with the piston (to the right) and with the stop (left of the 18 disk).

Figure 3 shows the wrapped-phase map corresponding to that set of phasestepped images. Every black-to-white step on that pattern corresponds to a $2 \pi$ increase

21 in displacement, what in this experiment corresponds to $0.53 \mu \mathrm{m}$ per fringe along the

$22 \mathrm{X}$ axis, and results in a total displacement of $4.77 \pm 0.1 \mu \mathrm{m}$ along the $\mathrm{X}$ axis. A Cosine 23 transform unwrapping algorithm [22] was applied to the wrapped-phase map and the 
1 result is shown on Figure 4. On this image the area of the disk in white colour

2 corresponds to zero displacement, while the area of the image in black corresponds to

3 the maximum displacement of $4.77 \pm 0.1 \mu \mathrm{m}$.

4 [figures 2,a,b,c should go about here]

[figure 3 should go about here]

[figure 4 should go about here]

\subsection{Numerical differentiation of the ESPI in-plane deformation map}

The ESPI in-plane deformation maps can be numerically differentiated in order to obtain maps of in-plane strain, $(\partial u / \partial x)$. With this aim the maps of in-plane ESPI obtained with the Brazilian disk were processed in MatLab ${ }^{\mathrm{TM}}$, by The Mathworks Inc., at the Wolfson School of Mechanical and Manufacturing Engineering, Loughborough University, and the results are shown on Figure 5. On the figure it can be appreciated how the distribution of in plane strain has an elliptical distribution along a horizontal lobe. This is in agreement with the results of the double beam mutually incoherent illumination interferometer, the results of our photoelasticity experiments, and also in agreement with the results by A. Castro-Montero et al. [21] obtained by holographic interferometry on a Brazilian disk.

[figure 5 should go about here] 
2 To complement the analysis, photoelasticity results were obtained by means of a

3 reflection polariscope (030-series by Measurements Group Inc., North Carolina,

4 USA) consisting of two polariser/quarter-wave assemblies mechanically coupled for

5 synchronous rotation. This optical set-up produces an isochromatic pattern that

6 highlights the distribution of pure in-plane strain.

7 The qualitative results of the photoelasticity experiments, figure 6 , were in

8 agreement with the distribution of in plane strain previously obtained by means of the

9 numerical differentiation of the in plane deformation maps, figure 5. The in plane

10 strain was concentrated on a central lobe orientated along the direction of the

11 application of the force. This result was also in agreement with the result of the dual

12 beam mutually incoherent illumination interferometer.

13

14

[Figures 6 a,b,c,d should go about here] 
2 The out-of-plane component of the strain, ( $\partial \mathrm{w} / \partial \mathrm{x})$, was analysed independently with

3 the specimen compressed by the same forces and rig used throughout the experiments.

4 The amount of horizontal shearing ( $\delta \mathrm{x})$ was equal to $5 \mathrm{~mm}$, and as in the case of ESPI,

5 a three images phase-step system was implemented to obtain quantitative results from

6 the experiments, Figure 7.

7

8

9

11 of non-equispaced vertical parallel fringes across the surface of the object. The change

12 in spatial frequency of the fringes along the surface of the disk is more evident as the

13 force increases. Figures 8-10 show the wrapped-phase map corresponding to forces of $14 \quad 17.5 \pm 0.1 \mathrm{Kg}$-Force, $20.0 \pm 0.1 \mathrm{Kg}$-Force and $22.5 \pm 0.1 \mathrm{Kg}$-Force respectively. As with 15 all the experiments presented in this work, the force is applied from the right hand 16 side of the image.

18 side and two more fringes) what indicates a maximum out-of-plane strain of $19 \quad 106.4 \pm 10.6 \mu$ strain on the left hand side of the disk. Figure 9 and Figure 10 show a 20 maximum out-of-plane strain of 159.6 $\pm 10.6 \mu$ strain and $212.8 \pm 10.6 \mu$ strain 21 respectively. [insert figure 8 about here] 
4.5. Dual beam mutually incoherent illumination interferometer analysis of the Brazilian Disk

This new interferometer uses two mutually incoherent beams making incidence at opposite angles from the optical axis of the observation system, where a Michelson

8 shearing head is used to introduce the lateral shifting between images. The speckle

9 fringe patterns predicted by the theoretical analysis will consist in the moiré-like inter-

10 crossing of the pure out-of-plane strain fringes with the pure in-plane strain fringes.

11 Moiré fringes will be visible in the zones of the object where the equations for out-of-

12 plane strain and in-plane strain are simultaneously satisfied, equations [20] and [21].

13 This represents an improvement from an interferometer with double beam coherent 14 illumination [23], where the moiré pattern also included the ESPI in-plane fringes.

The optical set-up was as described in figure 1, with the difference that instead 16 of illuminating with one laser source split into two beams with orthogonal 17 polarisations, we utilised two beams from two different laser sources with the same 18 wavelength. This ensured that no amount of depolarisation could reduce the contrast 19 of the fringes, since the beams were permanently incoherent to each other.

Figure 11 shows the reference image of the Brazilian disk with the two 21 laterally shifted images, $\delta x=10 \mathrm{~mm}$, overlapping on the image plane. A typical set of 22 results is shown in Figures 12 a) to d). 
3

4

[insert figure $12 \mathrm{e}$ ) about here]

5

Juan F Román et al. Page 21/43 Interferometer2 Roman et al v13.4.doc 
1 Figure 12 a) shows the specimen under just enough force to show only out-of-

2 plane strain on the object: the force was not enough to show a visible pattern of in-

3 plane strain and equation [21] for the in-plane strain corresponded to $\mathrm{m}=0$ across the

4 whole specimen. The pressure was then very slowly increased on the DH-Budenburg

5 dead-weight rig to show how the in-plane strain fringe comes up into view.

6 Figure $12 \mathrm{~b}$ ) shows the specimen after the force was increased, and now the 7 central part of the disk begins to show some decorrelation. This means that in the 8 central lobe the in-plane strain has increased up to the point where equation [21]

9 corresponds to $\mathrm{m}<1$, still not enough to originate a fringe but enough to produce 10 decorrelation on the moiré pattern.

11 Figure $12 \mathrm{c}$ ) shows the specimen after another increase in the force applied. Now 12 the in-plane strain on the specimen is enough to reach $m=1$ in equation [21], what 13 gives rise to an elliptical distribution of in-plane strain at the centre of the disk, as 14 predicted on the previous experiments and shown for indication in Figure $12 \mathrm{e}$ ).

15 Figure $12 \mathrm{~d}$ ) shows how the central lobe in the centre of the specimen is now 16 even bigger $(m \geq 1)$, surrounded by a region where the fringes disappear in the moiré $17(0<\mathrm{m}<1)$ and then become visible again, $\mathrm{m}=0$.

18 The qualitative set of results confirm the theoretical predictions for speckle 19 shearing interferometer with two mutually incoherent symmetrically incident beams, 20 making it possible for the first time to observe a distribution of pure in plane strain by 21 optical means and in real time. 


\section{5. CONCLUSIONS}

2 A variation on a conventional Michelson-shearing interferometer has been presented,

3 in this case with the use of double beam mutually incoherent symmetrical

4 illumination. The theoretical analysis allows the prediction of the fringe patterns

5 resultant from this novel interferometer in both stationary state and in the presence of

6 phase-stepping introduced within the Michelson shearing-head.

7 A series of experiments were performed on a Brazilian disk specimen utilising 8 conventional speckle shearing techniques, numerical differentiation of ESPI

9 displacement maps and photoelasticity techniques. All the experimental results

10 showed the same consistent results with the sample under the same forces.

11 Furthermore, the dual beam mutually incoherent illumination interferometer did show

12 the predicted distribution of in plane strain fringes, and in agreement with the

13 experimental results from the conventional speckle interferometers.

14 The novel interferometer can highlight pure in plane strain in one single 15 measurement and in real time, with the advantage that now a single experiment 16 produces a measurement instead of having to perform alternative measurements with 17 in-plane ESPI and conventional ESPSI, since the combination of results from two 18 interferometers always produces an increase in the error of the measurement.

\section{Acknowledgements}

21 Bayer Diagnostics is the current affiliation of Juan F. Román, however this work

22 was done during his $\mathrm{PhD}$ research under the direction of John R. Tyrer at the Wolfson 
1 School of Mechanical and Manufacturing Engineering, Loughborough University,

2 and Vicente Moreno, at the Universidade de Santiago de Compostela, Spain.

3

4 -END-

Juan F Román et al. Page 24/43 Interferometer2 Roman et al v13.4.doc 
3 [1] P.K. Rastogi (Editor), Digital speckle pattern interferometry and related techniques (John Wiley \& Sons Ltd, Chichester, 2001).

[2] J.A. Leendertz, and J.N. Butters, 'An image shearing speckle pattern interferometer for measuring bending moments', Journal of Physics E, 6, (1973), 1107-1110.

9

10 [3] Y.Y. Hung, and C.E. Taylor, 'Measurement of slopes of structural deflections 11 by speckle shearing interferometry', Experimental Mechanics, (1974), 281-285.

13 [4] W. Steinchen, and L. Yang, Digital Shearography: Theory and Application of Digital Speckle Pattern Shearing Interferometry (SPIE Press, Bellingham, 2003).

[5] W. S. Wan Abdullah, J. N. Petzing and J. R. Tyrer, 'Wave-front divergence: A source of error in quantified speckle shearing data', Journal of Modern Optics 48 (2001), 757-772. 
1 [6] J. M. Huntley, "Automated Fringe Pattern Analysis in Experimental Mechanics:

A Review", Journal of Strain Analysis for Engineering Design, 33 (2), (1998), 105-125.

[7] K.F. Wang, A.K. Tieu and E. B. Li, 'Influence of in-plane displacement and strain components on slope fringe distributions in double-aperture speckle wedge shearing interferometry', Optics \& Laser Technology 31, (1999), 549554.

[8] T. Santhanakrishnan, N. Krishna Mohan, P.K. Palaniswamy, and R. S. Sirohi, 'Various speckle interferometric configurations for contouring and slope change measurement', J. Instrum. Soc. India 271 (1997), 16-22.

[9] R. S. Sirohi (Editor), Speckle metrology (Marcel Dekker Inc., New York, 1993).

[10] P. K. Rastogi, 'Measurement of in-plane strains using electronic speckle and electronic speckle-shearing pattern interferometry', Journal of Modern Optics 438 (1996), 1577-1581.

[11] Y.Y. Hung, and J.Q. Wang, 'Dual-beam phase shift shearography for measurement of in-plane strains', Optics and Lasers in Engineering 24 (1996), 403-413. 
1 [12] H.A. Aebischer and S. Waldner, 'Strain distributions made visible with imageshearing speckle pattern interferometry', Optics and Lasers in Engineering 26 (1997) 407-420.

[13] R. Kästle, E. Hack, and U. Sennhauser, 'Multiwavelength shearography for quantitative measurements of two-dimensional strain distributions', Applied Optics 381 (1999), 96-100.

[14] J. R. Tyrer and J. N. Petzing, 'In-plane electronic speckle pattern shearing interferometry', Optics and Lasers in Engineering 26 (1997), 395-406.

[15] J. N. Petzing, and J. R. Tyrer, 'In-plane electronic speckle pattern shearing interferometry: a theoretical analysis supported with experimental results', paper presented at Interferometry'94, Warsaw, Poland, Proceedings of Interferometry'94, SPIE 2342 (1994), 27-36,.

[16] J. F. Román, J. N. Petzing, and J. R. Tyrer, 'Development of novel speckle shearing interferometers for direct in-plane strain measurement', Proceedings of FASIG'98, Institute of Physics, Applied Optics \& Optoelectronics Conference (UK, Brighton, April 1998).

[17] K. Patorski, 'Shearing interferometry and the moire method for shear strain determination', Applied Optics 2716 (1988), 3567-3572. 
1 [18] P. K. Rastogi, 'Direct determination of large in-plane strains using high resolution moiré shearography', Optics and Lasers in Engineering 29 (1998), 97-102.

[19] D. W. Robinson and G. T. Reid, Interferogram analysis: Digital fringe pattern measurement techniques (Bristol: IOP Publishing Ltd., 1993).

[20] J. W. Dally and W. F. Riley, Experimental stress analysis, (McGraw-Hill International Editions, New York, 1991).

[21] A. Castro-Montero, A. Jia and S. P. Shah, 'Evaluation of damage in Brazilian test using holographic interferometry', American Concrete Institute Materials Journal 923 (1995), 268-275.

[22] D. Kerr and A. Davila, 'Unwrapping of interferometric phase fringe maps using the discrete cosine transform', Applied Optics 35 (1996), 810-816.

[23] J. F. Román, V. Moreno, J. N. Petzing and J. R. Tyrer, 'Analysis of coherent symmetrical illumination for electronic speckle pattern shearing interferometry', Journal of Modern Optics 526 (2005), 797-812.

[24] D. I. Farrant and J. N. Petzing, 'Sensitivity errors in interferometric deformation metrology', Applied Optics 4228 (2003), 5634-5641. 


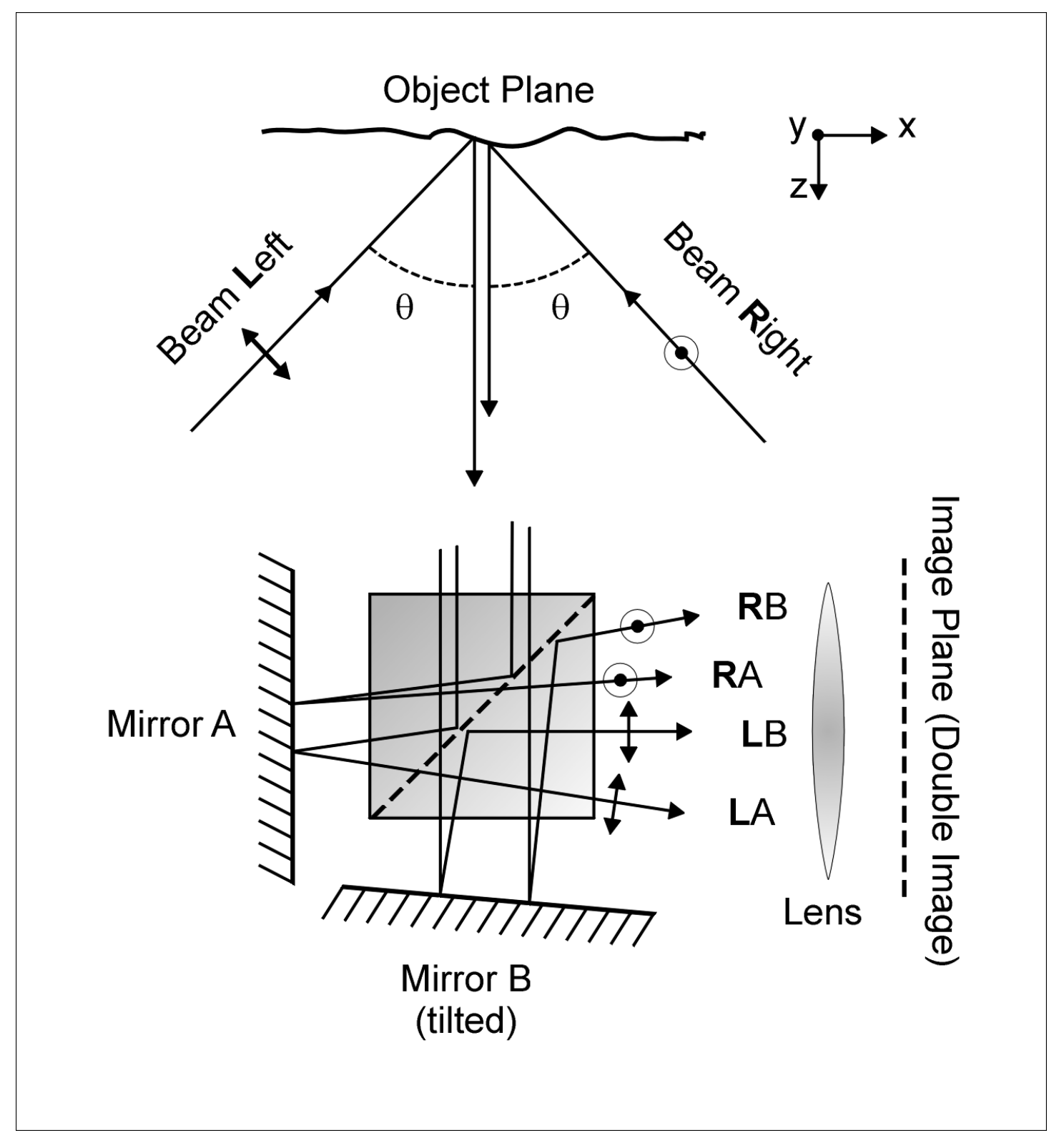

Figure 1. Speckle shearing interferometry with symmetrical mutually incoherent illumination. 


\begin{tabular}{|c|c|c|c|}
\hline Mirror & $\begin{array}{c}\text { Component of the } \\
\text { Displacement }\end{array}$ & $\begin{array}{l}\text { Optical Path Change } \\
\text { in Beam L }\end{array}$ & $\begin{array}{c}\text { Optical Path Change } \\
\text { in Beam } R\end{array}$ \\
\hline $\mathbf{A}$ & u (X axis) & $+u \cdot \operatorname{Sin} \theta$ & $-\mathrm{u} \cdot \operatorname{Sin} \theta$ \\
\hline $\mathbf{A}$ & v (Y axis) & nil & nil \\
\hline $\mathbf{A}$ & w ( $\mathrm{Z}$ axis) & $-\mathrm{w} \cdot(1+\operatorname{Cos} \theta)$ & $-\mathrm{w} \cdot(1+\operatorname{Cos} \theta)$ \\
\hline B & $\mathrm{u}+\delta \mathrm{u}(\mathrm{X}$ axis $)$ & $+(\mathrm{u}+\delta \mathrm{u}) \cdot \operatorname{Sin} \theta$ & $-(u+\delta u) \cdot \operatorname{Sin} \theta$ \\
\hline B & $\mathrm{v}+\delta \mathrm{v}(\mathrm{Y}$ axis $)$ & nil & nil \\
\hline B & $\mathrm{w}+\delta \mathrm{w}(\mathrm{Z}$ axis $)$ & $-(\mathrm{w}+\delta \mathrm{w}) \cdot(1+\operatorname{Cos} \theta)$ & $-(\mathrm{w}+\delta \mathrm{w}) \cdot(1+\operatorname{Cos} \theta)$ \\
\hline
\end{tabular}

Table 1. Optical path changes in double beam interferometer. 


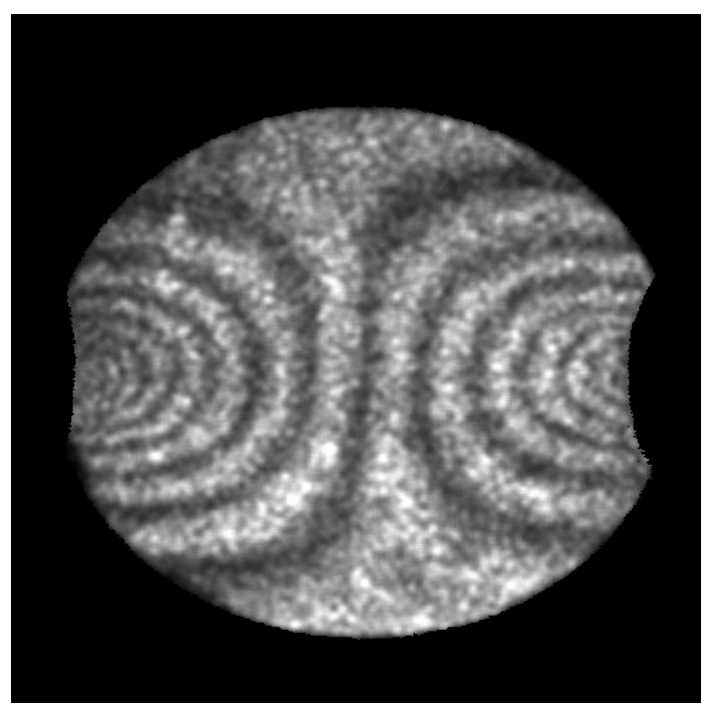

2.a

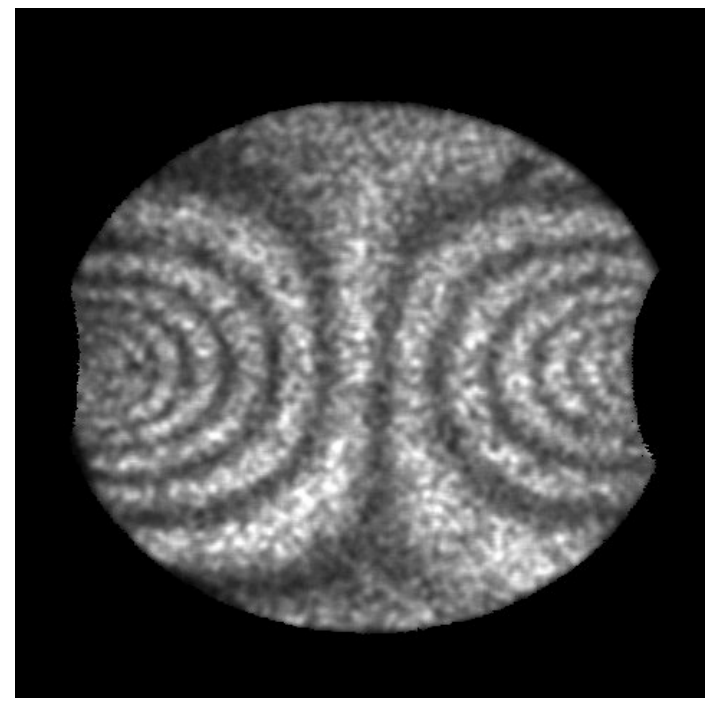

2.b

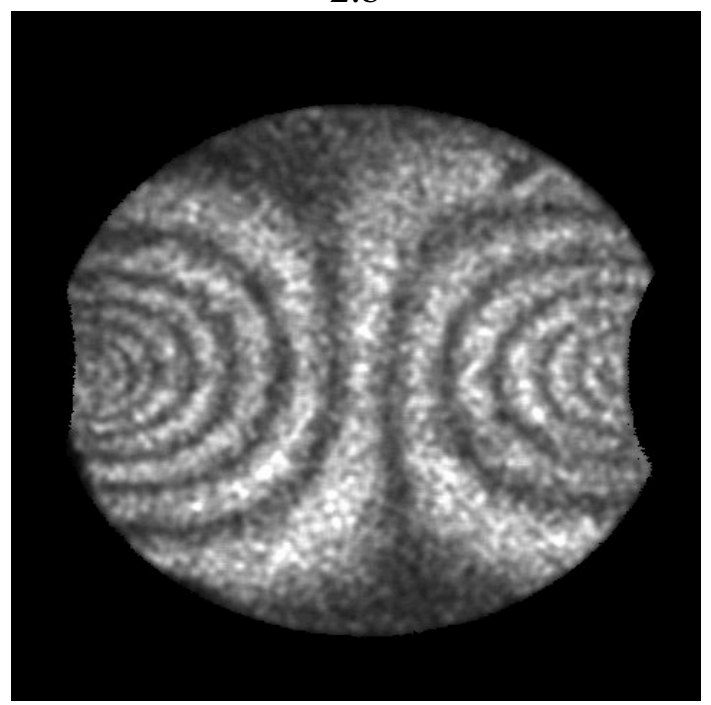

2.c

Figures 2 a,b,c). In Plane ESPI with horizontal illumination on the Brazilian disk, Force $=171.6 \pm 1$ Newton, a) $\phi=0, b) \phi=-2 \pi / 3$, c) $\phi=+2 \pi / 3$. 


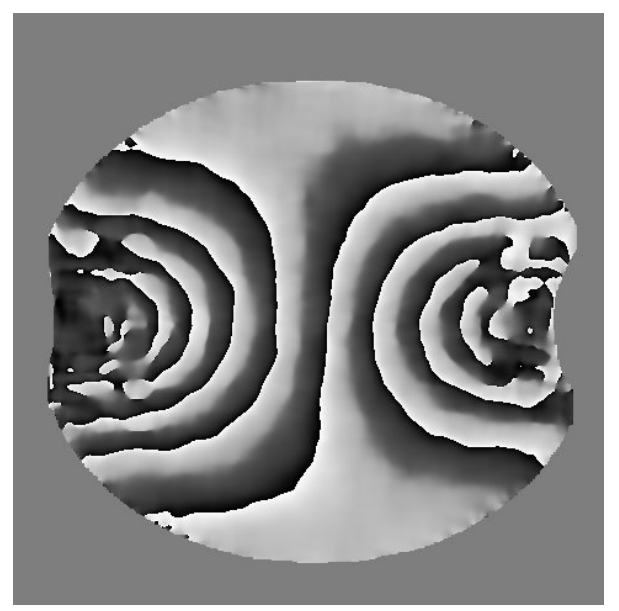

Figure 3. Wrapped phase map corresponding to Force $=171.6 \pm 1$ Newton.

Juan F Román et al. Page 32/43 Interferometer2 Roman et al v13.4.doc 


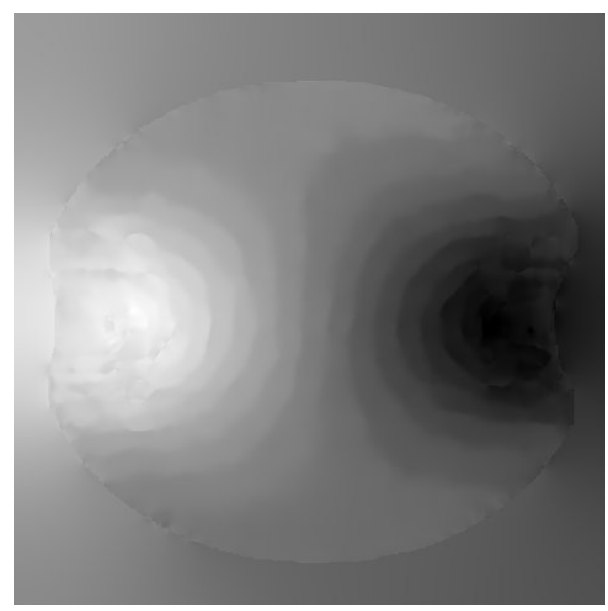

Figure 4. Unwrapped-phase map for Brazilian disk, Force $=171.6 \pm 1$ Newton.

Juan F Román et al. Page 33/43 Interferometer2 Roman et al v13.4.doc 


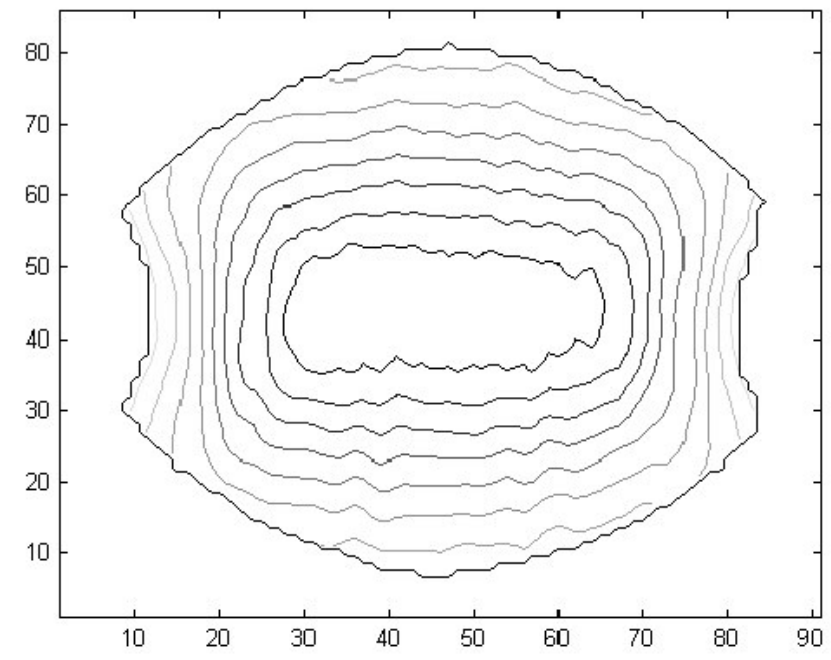

Figure 5. Contour map showing the distribution of pure in plane strain (maximum at the centre, arbitrary units), from the differentiation of the in plane displacement maps. 


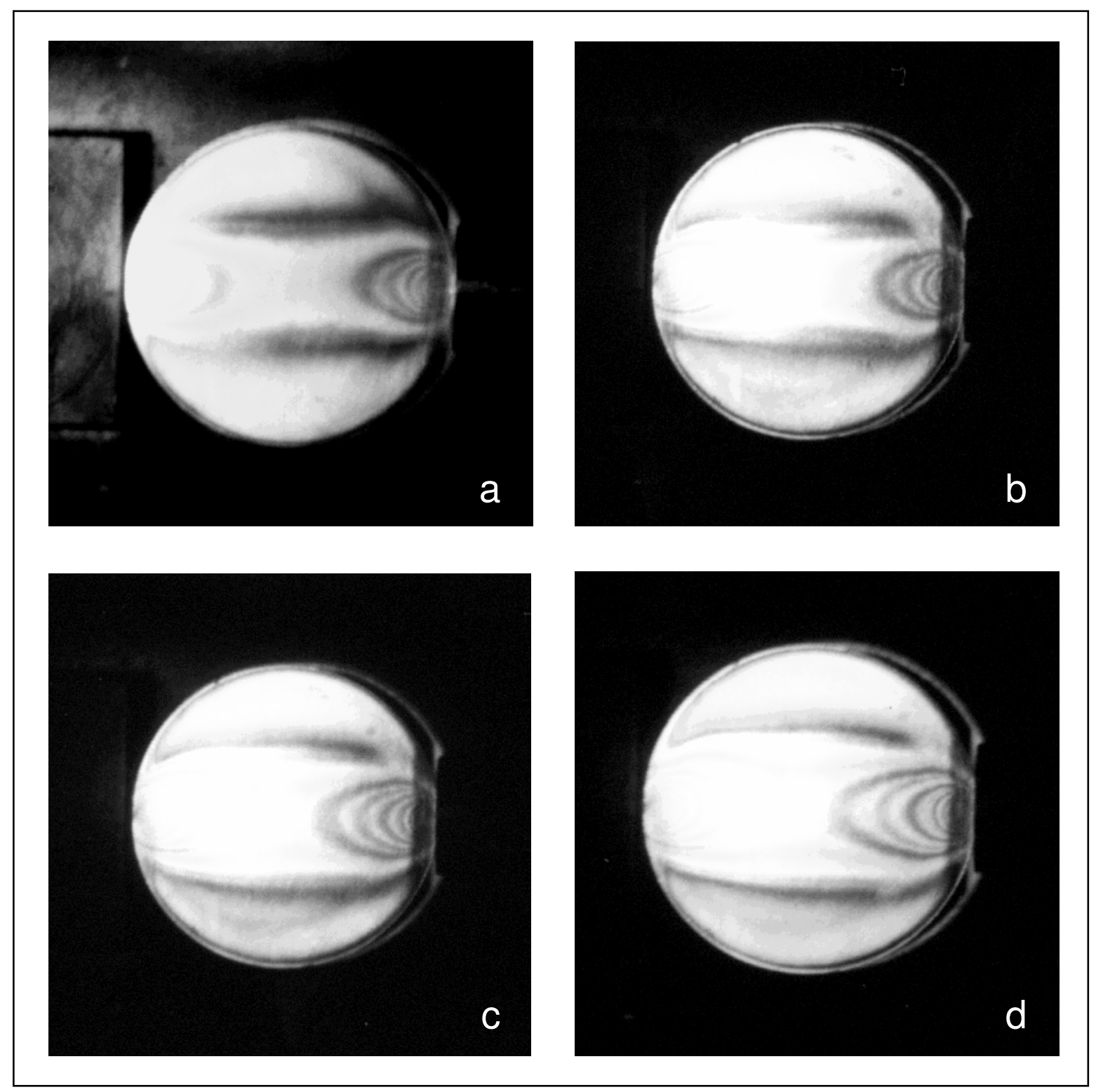

Figure 6, a) to d). Qualitative results from the photoelasticity experiments show a distribution of in-plane strain located inside a horizontal central lobe. 


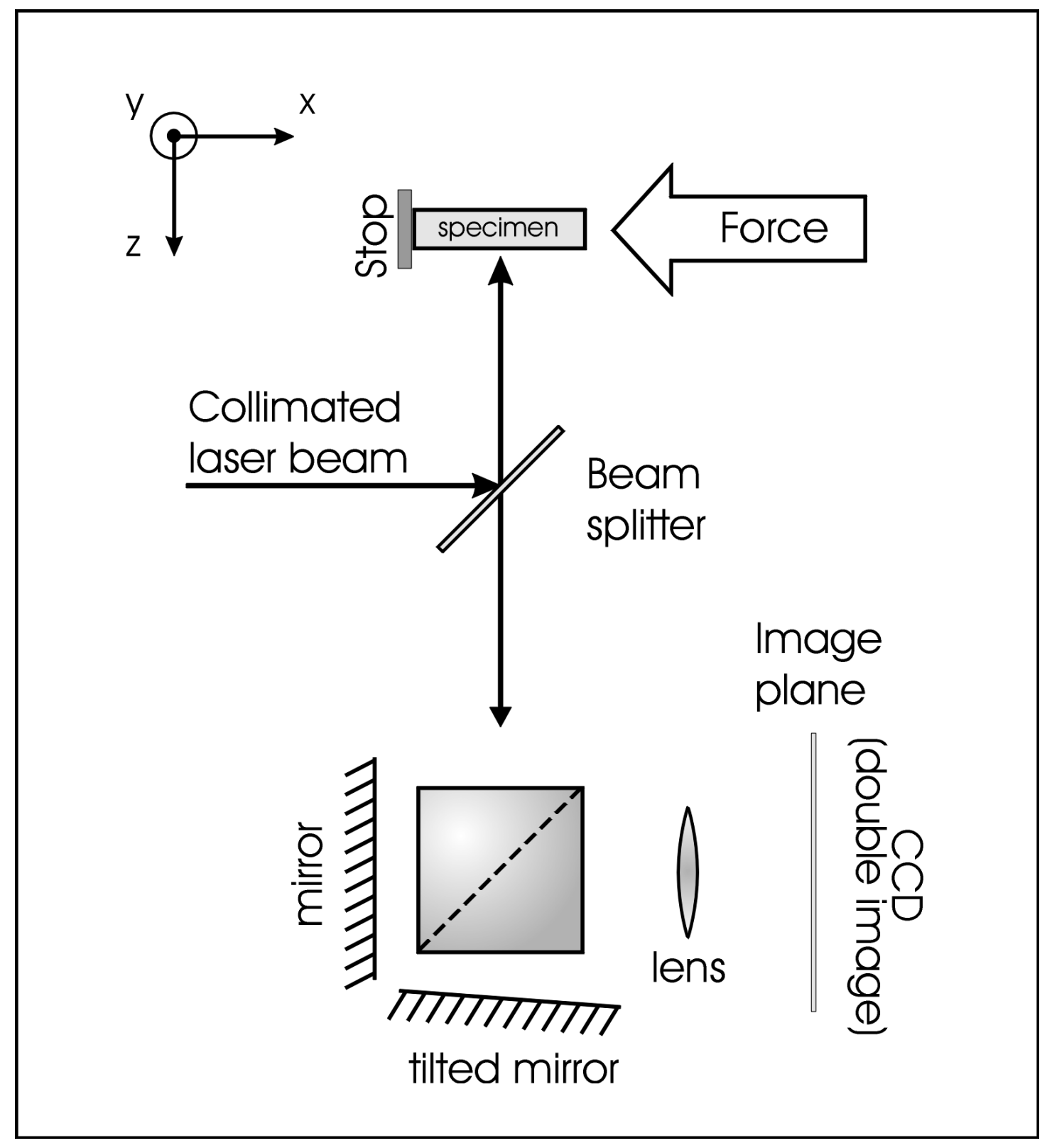

Figure 7. Optical set-up for out-of-plane ESPSI, with perpendicular illumination. 


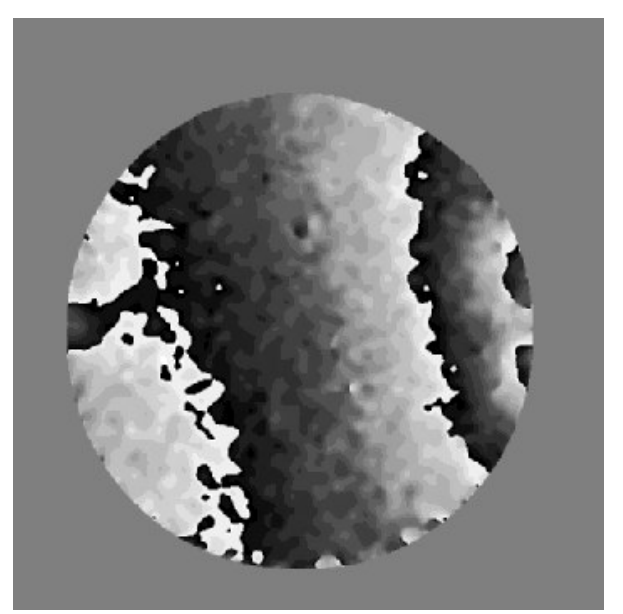

Figure 8. Wrapped-phase map of out-of-plane ESPSI with horizontal shear, showing fringes corresponding to the out of plane component $(\partial w / \partial x)$ of the strain, Force $=17.5 \pm 0.1 \mathrm{Kg}$-Force. 


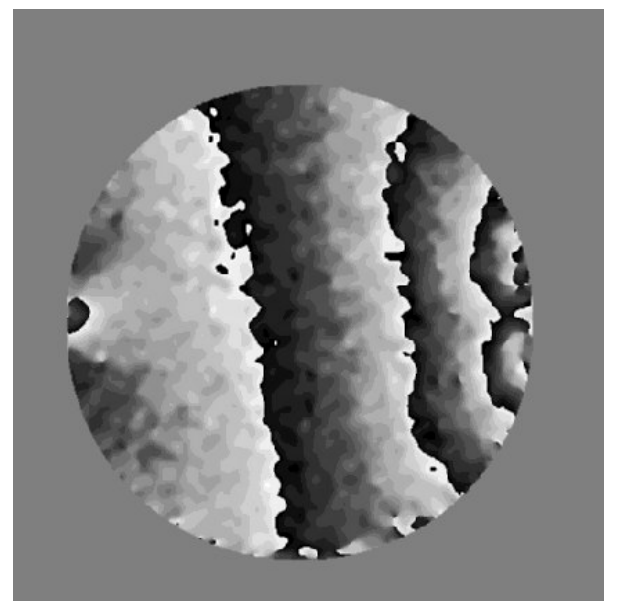

Figure 9. Same as previous, Force $=20.0 \pm 0.1 \mathrm{Kg}$-Force.

Juan F Román et al. Page 38/43 Interferometer2 Roman et al v13.4.doc 


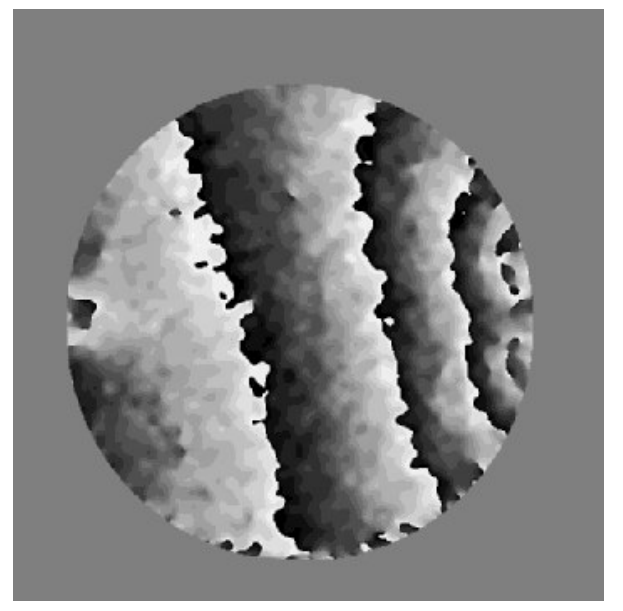

Figure 10. Same as previous, Force $=22.5 \pm 0.1 \mathrm{Kg}$-Force.

Juan F Román et al. Page 39/43 Interferometer2 Roman et al v13.4.doc 


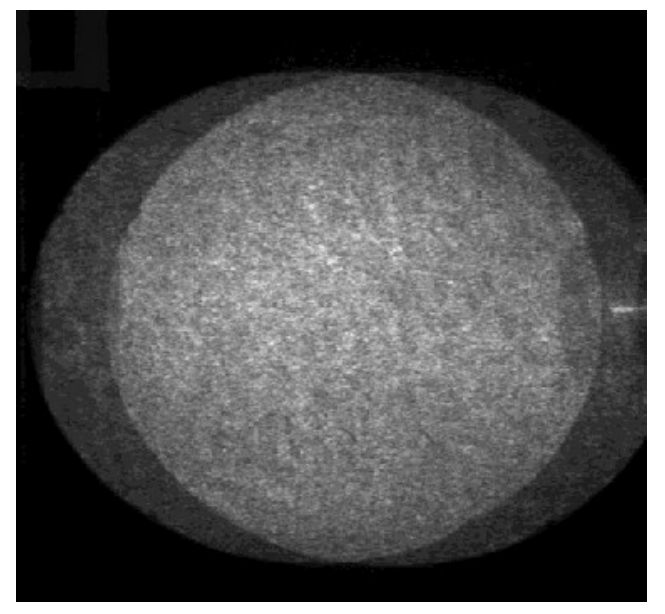

Figure 11. The reference image consists of two laterally shifted images.

Juan F Román et al. Page 40/43 Interferometer2 Roman et al v13.4.doc 


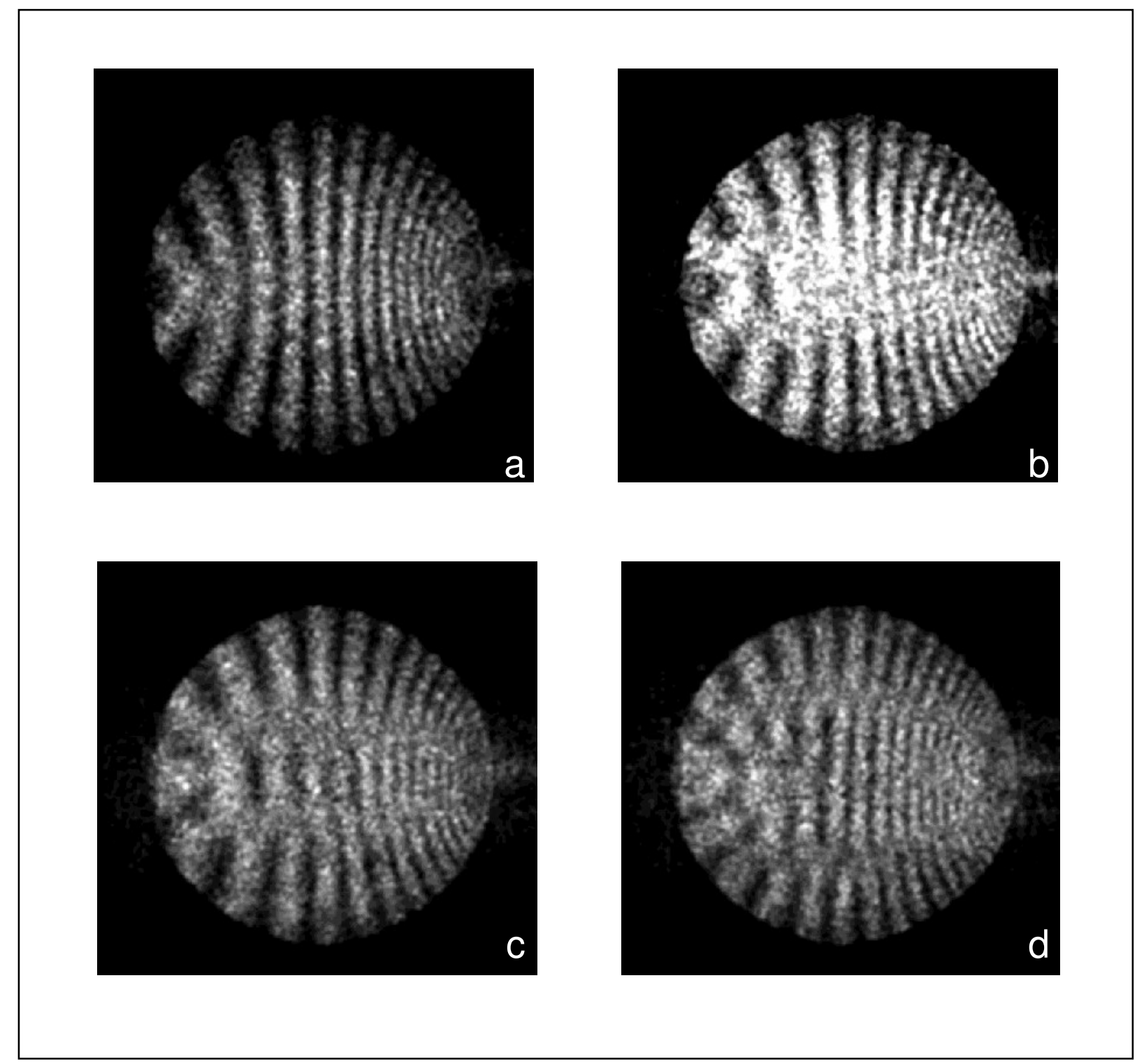

Figures 12 abcd. Distribution of in-plane strain is shown in real-time with a double beam mutually incoherent ESPSI interferometer. 


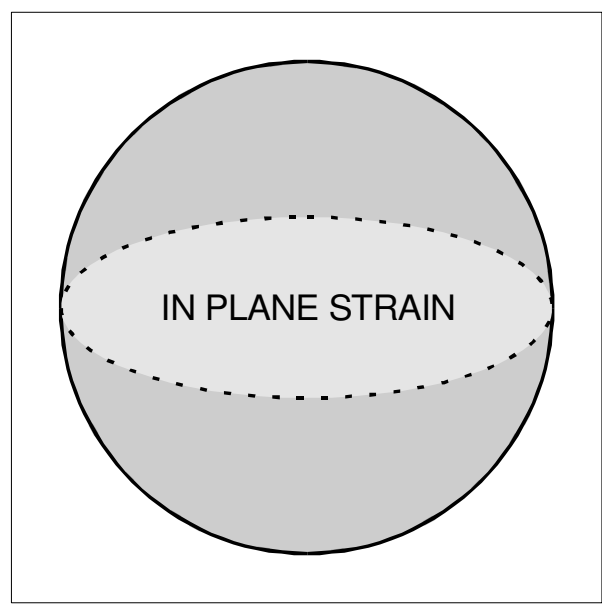

Figure 12 e). Diagram of the distribution of in-plane strain. 


\section{List of figure captions}

Figure 1. Speckle shearing interferometry with symmetrical mutually incoherent illumination.

Table 1. Optical path changes in double beam interferometer.

Figures 2 a,b,c). In Plane ESPI with horizontal illumination on the Brazilian disk, Force $=171.6 \pm 1$ Newton, a) $\phi=0, b) \phi=-2 \pi / 3, c) \phi=+2 \pi / 3$.

Figure 3. Wrapped phase map corresponding to Force $=171.6 \pm 1$ Newton.

Figure 4. Unwrapped- phase map for Brazilian disk, Force $=171.6 \pm 1$ Newton.

Figure 5. Contour map showing the distribution of pure in plane strain (maximum at the centre, arbitrary units), from the differentiation of the in plane displacement maps.

Figure 6, a) to d). Qualitative results from the photoelasticity experiments show a distribution of in-plane strain located inside a horizontal central lobe.

Figure 7. Optical set-up for out-of-plane ESPSI, with perpendicular illumination.

Figure 8. Wrapped-phase map of out-of-plane ESPSI with horizontal shear, showing fringes corresponding to the out of plane component $(\partial w / \partial x)$ of the strain, Force $=17.5 \pm 0.1 \mathrm{Kg}$-Force.

Figure 9. Same as previous, Force $=20.0 \pm 0.1 \mathrm{Kg}$-Force.

Figure 10. Same as previous, Force $=22.5 \pm 0.1 \mathrm{Kg}$-Force.

Figure 11. The reference image consists of two laterally shifted images.

figures 12 abcd. Distribution of in-plane strain is shown in real-time with a double beam mutually incoherent ESPSI interferometer.

Figure 12 e). Diagram of the distribution of in-plane strain.

END 